\title{
Influence de la durée de jeûne avant l'abattage et du poids à la mise en gavage des canards de Barbarie sur la composition chimique hépatique
}

\author{
C Baudonnet-Lenfant, A Auvergne, R Babilé
}

\author{
ENSAT, Laboratoire de zootechnie et des produits animaux, \\ 145 Av de Muret, 31076 Toulouse Cedex
}

(Reçu le 12 mars 1991; accepté le 26 juin 1991)

\begin{abstract}
Résumé - L'incidence du poids vif à la mise en gavage (format : 3,$66 ; 3,79$ et $3,92 \mathrm{~kg}$ ) et du délai de jeûne (13 et $19 \mathrm{~h}$ ) sur les performances zootechniques, sur la composition biochimique et le profil des acides gras totaux (AGT) du foie ont été étudiées sur 42 canards mâles de Barbarie à l'issue d'un gavage de 12,5 j. Pour les animaux de format moyen et lourd, l'allongement du délai de jeûne avant l'abattage entraîne une perte pondérale reflétant en partie la vidange du tractus digestif. Une diminution de $15 \%$ des poids des foies est enregistrée sans modification de leur constitution biochimique et du profil des acides gras. La quantité de la fraction indosée diminue avec l'allongement de la privation d'aliment pour les 3 formats. Le pourcentage de fonte, à 55 comme à $105^{\circ} \mathrm{C}$, est significativement plus important après $19 \mathrm{~h}$ de jeûne, ce qui pourrait être le signe d'une lipolyse accrue. Le poids vif et le poids saigné plumé restent différents entre les 3 formats. Au niveau du foie gras des animaux légers, si la production est peu différente, on observe des proportions plus importantes d'eau et de protéines, corrélativement à un taux d'indosé inférieur. Le profil AGT laisse apparaître des écarts entre les 2 formats extrêmes : les animaux légers présentent en particulier plus de C16:0 et moins de C18:0 et C18:2.
\end{abstract}

canard / format / jeûne / foie / acide gras

Summary - Effect of starvation before slaughter and size before force-feeding on fatty liver composition in Muscovy ducks. The biochemical composition of the liver and total fatty acid profile were recorded in 42 fatty livers of male Muscovy ducks after 12 1/2 d of force feeding. The effects of stanvation $\left(J_{1}: 13 \mathrm{~h}\right.$ and $J_{2}: 19 \mathrm{~h}$ ) and body size (FLe, light: $3,66 \mathrm{~kg} ; F M o$, middle : $3.79 \mathrm{~kg}$ and Flo, heavy: $3.92 \mathrm{~kg}$ ) before force-feeding were studied. A longer starvation period before slaughter induced losses resulting from the emptying of the digestive tract (330 vs $291 \mathrm{~g}$ ) for the FMo and FLo birds in particular. Fatty liver weights decreased by $=15 \%$, but no difference was noticed in their biochemical composition and fatty acid profile. The undefined amount decreased in the same manner in the 3 groups when the starvation period was increased (23.71 vs $15.77 \mathrm{~g}$ ). Technical melting estimated by liver weight losses after $55^{\circ} \mathrm{C}$ and $105^{\circ} \mathrm{C}$ heat treatment was significantly greater after $19 \mathrm{~h}$ starvation ( 8.68 vs $19.64 \%$ and 44.56 vs $54.04 \%$, respectively). This may be the result of increased lipolysis. Differences in body size between the 3 groups remained after force feeding. The livers of FLe ducklings were similar as regards weight and lipid percentage, but they exhibited a greater proportion of moisture or proteins, and correlatively a similar undefined amount. These birds presented significantly more C16:0 (34.7 vs $32.1 \%$ ) and less C18:0 (9.8 vs $11.9 \%$ ), C18:2 (0.9 vs $1.4 \%)$ than the heavier group. This difference might reflect a lower saturation point for the lightest animals. 


\section{INTRODUCTION}

La nécessité de respecter un certain délai de jeûne avant l'abattage des animaux est particulièrement liée à des considérations d'ordre sanitaire. La privation de nourriture permet d'assurer la vidange du tractus digestif, ce qui minimise les contaminations des carcasses par les bactéries fécales. Les études sur le transit ont montré que chez les volailles, nourries ad libitum, $80 \%$ de la ration étaient excrétés au bout de 8 à $12 \mathrm{~h}$ (Leclercq et al, 1989). Cependant, une surcharge alimentaire relative, distribuée par gavage, entraîne un ralentissement de la motricité du tube digestif proximal chez le poulet et par conséquent un allongement des temps de transit (Sibbald et Moorse, 1983).

Par ailleurs, l'incidence de la durée du jeûne sur le poids vif des animaux, les viscères et en particulier le foie, a été largement étudiée chez les mammifères tels que le rat (Burrin et al, 1988), mais aussi chez les oiseaux : l'oie (Le Maho et al, 1981), le poulet (Jensen et al, 1984), la dinde (Augustine, 1982) et le canard (Leclercq et al, 1989). II ressort de ces travaux que le jeûne alimentaire entraînait avant tout une diminution pondérale des animaux et que le foie semblait être le plus sensible. En effet, ce dernier est le site majeur de la lipogénèse et assure $90 \%$ de la synthèse de novo des acides gras libres chez les espèces aviaires (Leveillé et al, 1975). Une privation de nourriture abaisse le niveau de synthèse hépatique des lipides et les activités des enzymes impliquées dans ces processus (Goodridge, 1968; Goodridge et Ball, 1968; Yeh et al, 1970; Yeh et Leveillé, 1971).

Dans le cas des palmipèdes soumis au gavage, le délai de jeûne avant l'abattage peut avoir un intérêt particulier dans la mesure où les variations éventuelles du poids et de la qualité des foies gras ont une incidence directe sur la valorisation de ce pro- duit. II nous a donc paru intéressant d'estimer, chez des canards ayant subi un forçage alimentaire, l'incidence du jeûne sur l'engraissement hépatique au travers de la composition chimique de cet organe.

\section{MATÉRIELS ET MÉTHODES}

L'essai a été mené à partir de 180 canards de Barbarie mâles de type génétique R61, commercialisés par la société Grimaud frères. Ces animaux sont prélevés dans un lot d'élevage à l'âge de 9 semaines. Le tri a été effectué selon un critère pondéral afin d'obtenir un échantilion homogène centré sur un poids vif de $3 \mathrm{~kg}$.

A l'âge de 10 semaines, les canards sont pesés, bagués. Trois classes de poids sont constituées : les animaux les plus légers (FLe : $2858 \pm 59,11 \mathrm{~g}$ ), puis les animaux de poids moyen (FMo : $3023 \pm 31,66 \mathrm{~g}$ ) et les lourds (FLo : $3126 \pm 55,45 \mathrm{~g}$ ).

Pendant la période précédant le gavage (prégavage), l'ensemble des animaux reçoit un rationnement par repas, de $2 \mathrm{~h}$ une fois par jour. Afin d'améliorer les capacités d'ingestion, l'alimentation est libérée 3 j avant l'entrée en gavage (Babilé, 1989). L'aliment utilisé pendant le prégavage est un aliment type du commerce (tableau I). L'eau de boisson est distribuée à volonté.

A l'issue de cette période, les animaux âgés de 12 semaines sont pesés individuellement (PEG) après un jeûne minimum de $18 \mathrm{~h}$.

Quarante-deux canards mis en gavage à la station, sont répartis dans 6 épinettes de 7 animaux chacune. Ils reçoivent quotidiennement 2 repas de maïs étuvé, pendant 12,5 j, suivant le modèle décrit par Babilé et al (1987a). Afin de maîtriser l'incidence du délai de jeûne depuis le dernier repas de gavage sur les paramètres mesurés, 2 séquences d'abattage sont retenues, correspondant à un délai moyen de jeûne de $13 \mathrm{~h}\left(\mathrm{~J}_{1}\right)$ et $19 \mathrm{~h}\left(\mathrm{~J}_{2}\right)$.

Après un ressuyage de $24 \mathrm{~h}$, une dissection anatomique est réalisée selon la méthode standardisée WPSA (1984). De plus, le contenu de la partie antérieure du tractus digestif (œsophage + proventricule) a été mesuré.

Un premier prélèvement est effectué sur les foies gras au niveau du lobe droit afin de réaliser les analyses biochimiques. 
Tableau l. Composition centésimale et chimique de l'aliment fourni pendant le prégavage.

\begin{tabular}{lc}
\hline Constituants & $\%$ \\
\hline Maïs & 10,5 \\
Blé & 26 \\
Orge & 35 \\
Soja 48 & 3 \\
Pois & 22 \\
CMV & 3,5 \\
& \\
EM $1 \mathrm{kcal} / \mathrm{kg}$ & 725 \\
MAT g/kg & 147,0 \\
AA soufrés g/kg & 5,5 \\
Méthionine g/kg & 2,3 \\
Lysine g/kg & 7,1 \\
MG g/kg & 21,0 \\
Cellulose brute g/kg & 43,0 \\
Ca g/kg & 8,0 \\
P g/kg & 6,0 \\
& \\
\hline
\end{tabular}

1 Valeur calculée.

Un deuxième échantillon, de quelques g (7$8 \mathrm{~g}$ ) est prélevé au niveau de la pointe du lobe intermédiaire gauche des foies gras afin de réaliser un test de fonte lipidique (Babilé et $\mathrm{Au}$ vergne, 1986). Les échantillons prélevés au moment de l'éviscération sont immédiatement congelés et stockés à $-18^{\circ} \mathrm{C}$.

Les échantillons issus du premier prélèvement sont broyés sous azote liquide à l'aide d'un broyeur à billes. La détermination de la matière sèche et des cendres est réalisée selon les méthodes classiques (JOCE, 1971a, 1971b) et la détermination des protéines ( $\mathrm{N}$ Kjeldahl $\times 6,25$ ) par dosage de l'azote par minéralisation acide et détection colorimétrique (Verdouw et al, 1977). La fraction lipidique est extraite à froid par le mélange chloroforme-méthanol (2-1, V/V) (Folch et al, 1957) et estimée par pesée après évaporation du solvant. La détermination de la composition en acides gras des lipides totaux a été réalisée par chromatographie en phase gazeuse (chromatographe Hewlett Packard 5890) après saponification puis estérification des acides gras libérés selon la méthode AFNOR (NFT 60-233).
Les esters méthyliques sont séparés sur colonne capillaire FFAP en silice fondue (phase greffée PEG-TPA, $L=50 \mathrm{~m}, \emptyset=0,2 \mathrm{~mm}$ ), avec la programmation de température suivante : tempéralure initiale $150^{\circ} \mathrm{C}$, augmentation de $15^{\circ} \mathrm{C} / \mathrm{min}$ entre 150 et $200^{\circ} \mathrm{C}$, palier de $12 \mathrm{~min}$, de $5^{\circ} \mathrm{C} / \mathrm{min}$ entre 200 et $210^{\circ} \mathrm{C}$ palier de $5 \mathrm{~min}$, de $1,5^{\circ} \mathrm{C} / \mathrm{min}$ entre 210 et $220^{\circ} \mathrm{C}$, et palier à $220{ }^{\circ} \mathrm{C}$ de $11 \mathrm{~min}$, soit une durée totale d'analyse de $\mathbf{4 0}$ min pour chaque injection. La température du détecteur à ionisation de flamme est de $250^{\circ} \mathrm{C}$. Les acides gras obtenus ont une longueur de chaîne comprise entre 12 et 20 atomes de carbone.

À l'issue de l'ensemble des analyses réalisées, nous avons été amenés à estimer une fraction indosée correspondant en majeure partie à des composés d'origine glucidique.

La comparaison des résultats a été réalisée par l'analyse de variance à 2 facteurs croisés (effet "format" des animaux, effet délai de jeûne) (Dagnélie, 1975).

\section{RÉSULTATS}

Les poids vifs à l'entrée en gavage (PEG) diffèrent significativement, conformément au protocole (FLe, FMo et FLo) et sont équivalents dans les 3 classes de poids selon le délai de jeûne $\left(J_{1}\right.$ et $\left.J_{2}\right)$ (tableau II).

Les poids vifs enregistrés après gavage restent statistiquement différents entre les 3 classes d'animaux (4 762 vs 4926 vs $5052 \mathrm{~g}$; FLe, FMo et FLo respectivement, $P<0,001$ ), avec un gain de poids moyen semblable au cours du gavage. L'observation des valeurs des poids-saignés-plumés (PSP) montre qu'il n'y a plus d'écart significatif entre les animaux FMo et FLo; les animaux les plus légers (FLe), restent par contre différents des 2 autres classes.

L'incidence du délai de jeûne avant l'abattage apparaît nettement sur les valeurs enregistrées, et plus particulièrement pour les animaux FMo et FLo. L'allongement de la durée de privation de nourriture 
Tableau II. Évolutions pondérales et consommation au cours du gavage pour les 2 facteurs étudiés.

$$
\text { FLe FMo FLo flo } \stackrel{J_{1}}{J_{2}} \text { FMo flo } S \text { Format Jeûne Inter }
$$

$\begin{array}{lccccccccccc}\text { PEG } & 3697^{\mathrm{ab}} & 3826^{\mathrm{bcd}} 3944^{\mathrm{d}} & 3649^{\mathrm{a}} & 3784^{\mathrm{abc}} & 3897^{\mathrm{cd}} & 132,5 & * * * & \text { ns } & \text { ns } \\ \text { Gain Poids } & 1064^{\mathrm{ab}} & 1214^{\mathrm{b}} & 1180^{\mathrm{b}} & 1114^{\mathrm{ab}} & 1008^{\mathrm{a}} & 1093^{\mathrm{ab}} & 141,8 & \mathrm{~ns} & 10 & \mathrm{~ns} \\ \text { P vif Abat } & 4761^{\mathrm{a}} & 5040^{\mathrm{b}} & 5124^{\mathrm{b}} & 4763^{\mathrm{a}} & 4792^{\mathrm{a}} & 4990^{\mathrm{b}} & 160,0 & * * * & * & \text { ns } \\ \text { PSP } & 4240^{\mathrm{a}} & 4464^{\mathrm{b}} & 4481^{\mathrm{b}} & 4187^{\mathrm{a}} & 4240^{\mathrm{a}} & 4338^{\mathrm{ab}} & 166,5 & * & * & \text { ns } \\ \text { Intestin + més } & 310,4^{\mathrm{ab}} & 335,0^{\mathrm{b}} & 343,2^{\mathrm{b}} & 287,5^{\mathrm{a}} & 288,4^{\mathrm{a}} & 296,9^{\mathrm{a}} & 32,6 & \mathrm{~ns} & * * & \mathrm{~ns} \\ \text { Contenu Alt } & 28,4^{\mathrm{c}} & 27,8^{\mathrm{c}} & 10,8^{\mathrm{bc}} & 2,9^{\mathrm{abc}} & 0,0^{\mathrm{a}} & 1,3^{\mathrm{ab}} & 2,1 & \mathrm{~ns} & * & \text { ns } \\ \text { Consommation } & 6168 & 6286 & 6438 & 6048 & 6123 & 6415 & - & - & \text { non testé } & -\end{array}$

Difference statistique : ${ }^{* * *} P<0,001,{ }^{* *} P<0,01,{ }^{*} P<0,05,10 P<0,10$, ns : non significatif. Les moyennes affectées du même exposant sur une me̊me ligne ne diffèrent pas entre elles $(P>0,05) ; J_{1}$ : jeûne de $13 h ; J_{2}$ : jeûne de $19 \mathrm{~h}$; FLe : format léger; FMo : format moyen, FLo : format lourd; Inter : interaction entre les 2 facteurs étudiés; PEG : poids à l'entrée gavage; P vif Abat : Poids vif à l'abattage; PSP : poids saigné plumé; Intestin + més : intestin et mésentère; Contenu Alt : contenu du réservoir digestif antérieur (œsophage + proventricule). Analyse de variance réalisée sur les valeurs logarithmiques.

induit une perte de poids globale que l'on observe au niveau du poids vif (4978 vs $4851 \mathrm{~g}, \mathrm{~J}_{1}$ et $\mathrm{J}_{2}$ respectivement; $\left.P<0,05\right)$ et des gains de poids ( 1156 vs $1075 \mathrm{~g}$ respectivement). De même, le poids saigné plumé diminue de $143 \mathrm{~g}$ (4399 vs $4256 \mathrm{~g}$ respectivement, $P<0,05)$ entre $13 \mathrm{~h}$ et $19 \mathrm{~h}$. Cet écart est expliqué en partie par le poids de la masse intestinale (+ mésentère) qui décroît de $330 \mathrm{~g}$ à $291 \mathrm{~g}$ $(P<0,01)$. Le contenu du réservoir digestif antérieur évolue dans le même sens $\langle 3,07$ vs $0,73 \mathrm{~g}, \mathrm{~J}_{1}$ et $\mathrm{J}_{2}$ respectivement, $P<0,05)$. Si les animaux de format moyen ou lourd répondent significativement par des modifications pondérales à l'allongement de la durée de privation de nourriture; par contre, les canards plus légers présentent les mêmes valeurs.

Aucune interaction entre les 2 facteurs étudiés n'apparaît pour ces différents paramètres.
Les consommations par lots enregistrées au cours du gavage, bien que non testées statistiquement, semblent proportionnelles au format des animaux.

Les moyennes de poids de foie gras enregistrées pour chaque classe d'animaux à l'issue du gavage (tableau III) font apparaître, bien que cela ne soit statistiquement pas différent en valeur absolue, une meilleure performance pour les animaux les plus légers. Le rendement en foie gras exprimé en \% du PSP étant plus élevé pour ces derniers $(9,87$ et 9,38 vs $8,93 \%$; FLe, FMo et FLo, respectivement). Leurs foies gras renferment statistiquement plus d'eau et de minéraux que ceux des 2 autres types d'animaux, $(132,16 \mathrm{~g}$ vs $116,50 \mathrm{~g}$; FLe et FMo-FLo respectivement; $P<0,05$ pour l'eau et $1,74 \mathrm{~g}$ vs $1,47 \mathrm{~g}$; FLe et FMo-FLo respectivement; $P<0,10$ pour les minéraux). Cependant, les canards de format moyen (FMo) présentent 
Tableau III. Incidence des 2 facteurs étudiés sur les teneurs et pourcentages des constituants biochimiques du foie.

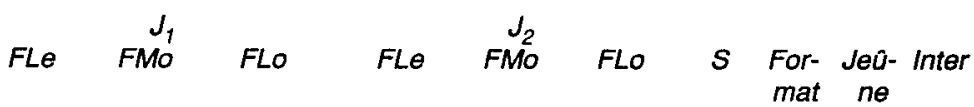

\begin{tabular}{|c|c|c|c|c|c|c|c|c|c|c|}
\hline Foie $(g)$ & $400,85^{a}$ & $442,03^{a}$ & $418,42^{a}$ & $424,04^{a}$ & $366,68^{b}$ & $366,31^{b}$ & 65,10 & ns & 10 & ns \\
\hline Fonte $55^{\circ} \mathrm{C}(\%)$ & $8,16^{a}$ & $11,67^{a b}$ & $5,71^{a}$ & $19,28^{b}$ & $23,21^{b}$ & $16,93^{\mathrm{b}}$ & 6,71 & ns & **** & ns \\
\hline Fonte $105^{\circ} \mathrm{C}(\%)$ & $47,33^{a b}$ & $47,23^{a b}$ & $38,67^{a}$ & $59,17 \mathrm{bc}$ & $52,44^{b c}$ & $50,29 \mathrm{bc}$ & 10,30 & ns & * & ns \\
\hline Humidité $(\mathrm{g})$ & $130,30^{\mathrm{bc}}$ & $128,87^{b c}$ & $121,01 \mathrm{abc}$ & $133,75^{c}$ & $104,78^{a}$ & $110,32^{\mathrm{ab}}$ & 19,13 & * & 10 & ns \\
\hline Protéines (g) & 25,60 & 24,82 & 25,49 & 25,58 & 21,88 & 24,12 & 3,29 & ns & ns & ns \\
\hline Lipides (g) & 225,93 & 253,91 & 250,72 & 253,93 & 214,58 & 214,84 & 49,09 & ns & ns & ns \\
\hline Minéraux $(\mathrm{g})$ & $1,91^{b}$ & $1,71^{\mathrm{ab}}$ & $1,42^{a}$ & $1,59^{a b}$ & $1,34^{a}$ & $1,37^{a}$ & 0,42 & 10 & 10 & ns \\
\hline Indosé (g) & $17,11^{a b}$ & $32,73^{c}$ & $19,77^{b}$ & $8,74^{a}$ & $24,10^{\text {bc }}$ & $15,67^{\mathrm{ab}}$ & 8,92 & $* * *$ & $*$ & ns \\
\hline \multicolumn{11}{|c|}{ En \% du poids de foie frais } \\
\hline Humidité & 32,51 & 29,33 & 28,90 & 31,87 & 29,53 & 30,38 & 4,42 & ns & ns & ns \\
\hline Protéines & 6,44 & 5,76 & 6,10 & 6,14 & 6,14 & 6,66 & 1,00 & ns & ns & ns \\
\hline Lipides & 56,28 & 57,32 & 59,96 & 59,22 & 57,39 & 58,30 & 5,31 & ns & ns & ns \\
\hline Minéraux & 0,47 & 0,40 & 0,34 & 0,39 & 0,38 & 0,38 & 0,12 & ns & ns & ns \\
\hline Indosé & $4,30^{a b}$ & $7,20^{d}$ & $4,71^{b c}$ & $2,26^{a}$ & $6,56^{\mathrm{cd}}$ & $4,29 a b$ & 1,83 & $\star \star \star \star$ & ns & ns \\
\hline \multicolumn{11}{|c|}{ En \% du poids de foie délipidé } \\
\hline Humidité & $74,50 \mathrm{bc}$ & $68,60^{a}$ & $72,21^{\mathrm{ab}}$ & $78,38^{\circ}$ & $68,60^{\mathrm{a}}$ & $72,92^{a b}$ & 3,84 & **** & ns & ns \\
\hline Protéines & $14,74^{\text {ab }}$ & $13,49^{a}$ & $15,24^{\text {ab }}$ & $15,19^{a b}$ & $14,53^{a b}$ & $15,88^{b}$ & 1,65 & 10 & ns & ns \\
\hline Minéraux & 1,07 & 0,93 & 0,85 & 0,96 & 0,89 & 0,89 & 0,24 & ns & ns & ns \\
\hline Indosé & $9,68^{a b}$ & $16,97^{\mathrm{c}}$ & $11,70^{\mathrm{bc}}$ & $5,36^{a}$ & $15,98^{\mathrm{c}}$ & $10,31^{b}$ & 4,34 & **** & ns & ns \\
\hline
\end{tabular}

Difference statistique : *** $P<0,001,{ }^{* *} P<0,01,{ }^{*} P<0,05,10 P<0,1$, ns : non significative. Les moyennes affectées du même exposant sur une même ligne ne diffèrent pas entre elles $(P<0,05)$. $\mathrm{J}_{1}$ : jeûne de $13 \mathrm{~h} ; \mathrm{J}_{2}$ : jeûne de $19 \mathrm{~h}$; FLe : format léger, FMo : format moyen; FLo : format lourd; Inter : interaction enire les 2 facteurs étudiés.

une quantité de résidu indosé plus élevé par rapport aux 2 autres groupes $(28,75$ et $15,08 \mathrm{~g}$ respectivement, $P<0,001$ ), différence qui apparaît aussi quand on exprime cette variable en pourcent du poids de foie (6,91 vs 3,84\%, FMo et FLe-FLo respectivement, $P<0,001$ ).

La différenciation des 3 groupes d'animaux se confirme lorsque les constituants biochimiques du foie sont exprimés en \% du tissu frais délipidé. Les animaux les plus légers ont une proportion d'eau hépatique plus importante que les 2 autres formats. Par contre, la fraction d'indosé est plus faible pour ces derniers, les animaux FMo présentant la teneur la plus élevée pour cet élément. Mais en ce qui concerne la teneur en matières azotées totales, ce sont les canards les plus lourds qui auraient tendance à avoir la plus forte proportion. La quantité de lipides, exprimée en valeur absolue ou en $\%$ du foie gras frais, est identique quel que soit le format des animaux et leurs performances.

Les poids de foies gras des animaux FMo et FLo diminuent avec l'allongement de la durée de jeûne $(P<0,10)$, alors que pour les canards FLe aucune modification 
pondérale au niveau hépatique n'est observée. Par contre, les quantités de lipides hépatiques ne sont pas sensibles à ce facteur et restent proportionnelles aux poids de foies gras quel que soit le format des animaux.

La quantité d'indosé du foie diminue avec le prolongement du jeûne $(23,71$ vs $15,77 \mathrm{~g}, \mathrm{~J}_{1}$ et $\mathrm{J}_{2}$ respectivement, $P<$ $0,05)$. De plus, les quantités d'eau et de minéraux hépatiques après $19 \mathrm{~h}$ de jeûne ont tendance à être plus faibles $(126,84$ vs $116,86 \mathrm{~g}, \quad J_{1}$ et $J_{2}$ respectivement $P<$ 0,10 pour l'eau et 1,68 vs $1,44 \mathrm{~g}, \mathrm{~J}_{1}$ et $\mathrm{J}_{2}$ respectivement $P<0,05$ pour les minéraux).

Les pourcentages de fonte mesurés à 55 et à $105^{\circ} \mathrm{C}$ sont significativement plus élevés pour les 3 groupes d'animaux dans la $2^{\mathrm{e}}$ série d'abattage $\left(8,68\right.$ vs $19,64 \%, J_{1}$ et $\mathrm{J}_{2}$ respectivement, $P<0,001$ à $55^{\circ} \mathrm{C}$ et 44,56 vs $54,04 \%, J_{1}$ et $J_{2}$ respectivement, $P<0,05$ à $105^{\circ} \mathrm{C}$ ).

L'analyse des profils d'acides gras totaux hépatiques (figs $1 a$ et $b$ ) fait apparaître globalement un effet significatif lié au format des animaux, non significatif en ce qui concerne le délai de jeûne et sans interaction entre les 2 effets testés. Les foies des canards FLo ont des proportions plus élevées pour la plupart des acides gras mineurs (C14, C16:1, C18:2 et C20) par rapport aux 2 autres lots. Ils présentent par ailleurs des taux d'acides palmitique et oléique légèrement plus faibles et un taux d'acide stéarique plus élevé en particulier par rapport aux animaux FLe. Enfin, quel que soit le délai de privation de nourriture, chaque format réagit dans le même sens.

\section{DISCUSSION ET CONCLUSION}

À l'issue de la période de gavage, les canards de format moyen et lourd semblent présenter un comportement identique entre les 2 séries d'abattage. L'allongement de la durée du jeûne avant l'abattage de ces animaux se traduit par une perte de poids vif et du poids-saigné-plumé, minimisant ainsi le gain de poids enregistré sur la période. Ce phénomène est lié en partie à la vidange du tractus digestif antérieur et postérieur, qui semble donc persister audelà de $13 \mathrm{~h}$ après le repas. Si, pour les canards destinés à la découpe de viande maigre, un délai de $8 \mathrm{~h}$ de jeûne est considéré comme suffisant pour leur permettre d'excréter la majeure partie de la fraction indigestible de l'aliment (Leclercq et al, 1989), en revanche, dans le cas d'animaux soumis à une suralimentation forcée, un délai plus important paraît donc nécessaire et cela essentiellement en raison du ralentissement de la motricité du tractus digestif antérieur lié à la surcharge alimentaire, comme l'ont déjà signalé Sibbald et Morse (1983) chez le poulet. Les animaux ayant ingéré des quantités de maïs proportionnelles à leur format, la diminution des poids de foie observée chez les animaux FMo et FLo, après $19 \mathrm{~h}$ de jeûne, peut être attribuée à l'allongement du délai d'abattage. Cette hypothèse s'appuie sur les liaisons déjà établies entre la consommation des animaux soumis au gavage et la quantité de foie gras obtenue d'une part ( $r=0,7$; Babilé, 1989), et le format des animaux d'autre part (Babilé et al, 1987b). Ces faibles variations du poids de foie observées entre les 2 périodes de jeûne n'ont aucune incidence sur la proportion relative d'un élément particulier intervenant dans la composition biochimique du foie, laissant supposer que cet organe présente les mêmes orientations métaboliques au cours des 2 stages de jeûnes retenus, et pour ces 2 groupes d'animaux. Comme l'avaient déjà observé Leclercq et Blum (1975) chez l'oie, la lipogenèse hépatique serait maintenue à un niveau élevé plusieurs $h$ après le dernier repas, principalement à la suite 
2
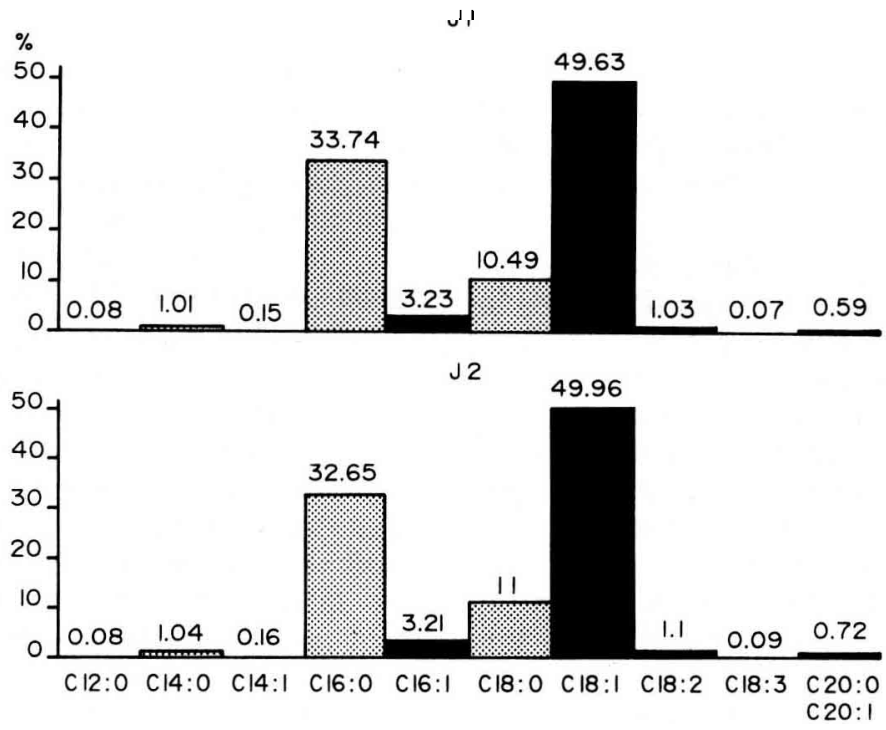

b \%
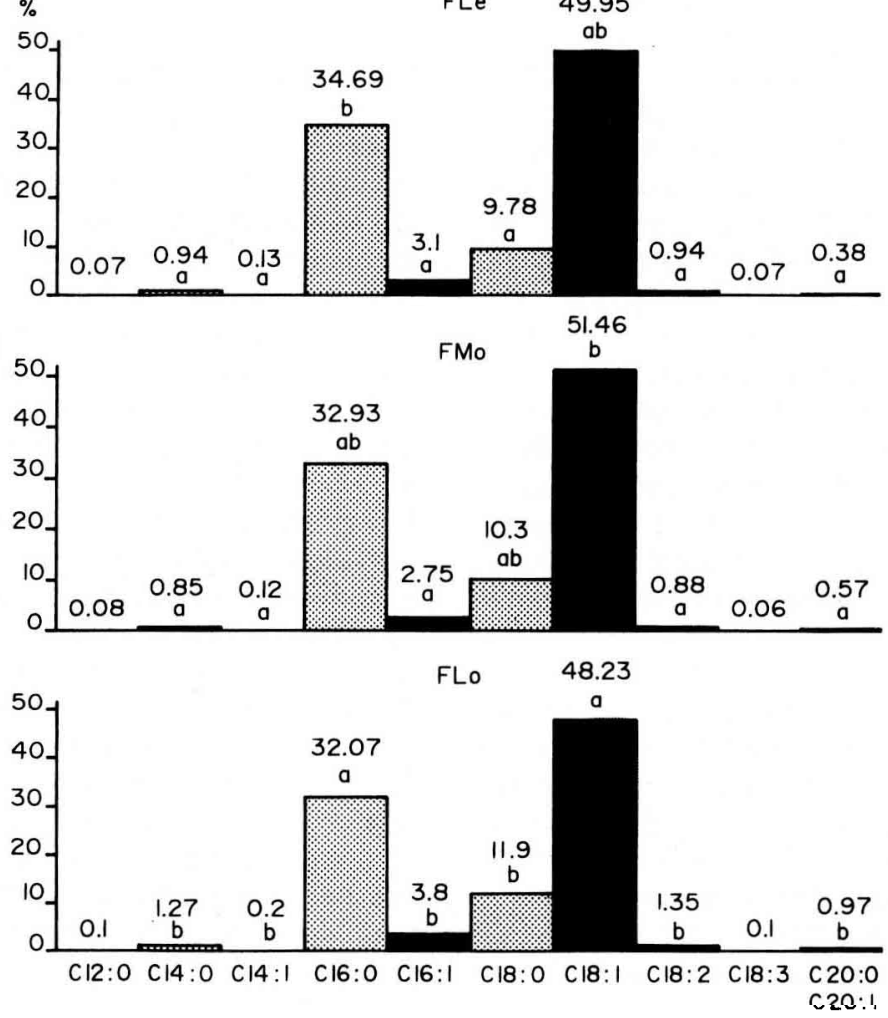

Fig 1. a) Incidence du jeûne $\left(J_{1}: 13 \mathrm{~h}, J_{2}: 19 \mathrm{~h}\right)$ sur le profil des acides gras totaux. b) Incidence du format (FLe : Léger, FMo : Moyen, FLo : Lourd) sur le profil des acides gras totaux. $\square$ Ac gras sat; $\square$ Ac gras insat. 
de l'augmentation de la quantité ingérée et du ralentissement de la motricité gastrique, provoquant des états digestifs plus longs.

Par contre, il semblerait que des animaux plus légers, ne réagissent pas de la même façon à un jeûne prolongé. Ainsi, le poids vif, le poids-saigné-plumé et le poids de foie des animaux restent-ils relativement stables. L'allongement du délai de privation de nourriture induit chez les animaux de petit format une diminution qui porte seulement sur la masse alimentaire aú niveau du tractus digestif antérieur. $\mathrm{Ce}$ processus semble lié à un état digestif encore très actif chez ces animaux et non à une différence de consommations puisqu'elles sont du même ordre de grandeur. Cependant, il n'y a pas de modification pondérale de l'intestin (+ mésentère) comme on peut l'observer pour les 2 autres formats. II semblerait donc que la surcharge alimentaire imposée par le gavage aux canards FLe provoque un état de saturation et consécutivement un ralentissement des phénomènes digestifs plus important que chez les 2 autres groupes d'animaux. Entre les 2 périodes et pour un poids de foie équivalent, on peut noter des différences de composition hépatique liées essentiellement à un accroissement de la fraction lipidique. II semble donc que pour ces animaux, le foie soit encore en phase active de synthèse de novo. De plus, cette augmentation s'oppose essentiellement à la chute de la teneur en indosé, qui pourrait être le reflet d'une importante activité de conversion des sucres de réserve en lipides. Outre le fait que la consommation en gavage soit liée au format (Babilé et al, 1987b), les animaux de petit format intrapopulation répondent plus vite à une stimulation alimentaire en gavage mais ne sembleraient pas supporter avec la même efficacité le prolongement de la durée du gavage (Babilé et al, 1987a). Cela suggère que les mécanismes et le métabo- lisme impliqués, responsables de la mobilisation et du transport des lipides du foie vers les tissus adipeux de la carcasse, ne s'adaptent pas aussi rapidement que la capacité de synthèse des lipides, ou que leur niveau de saturation est atteint.

Finalement, l'allongement de la durée de jeûne semble déprécier la qualité du foie gras, et ce quel que soit l'état physiologique des animaux à l'issue du gavage. II induit, dans les conditions d'un test de laboratoire, un pourcentage de fonte plus important qui pourrait être le reflet d'une lipolyse (ante mortem) plus active. L'examen du profil des acides gras hépatiques ne permet pas d'expliquer ces variations de qualité. La part importante représentée par les acides palmitique, oléïque et stéarique, de même que la teneur particulièrement faible en acide linoléiqque signent la prédominance d'une synthèse in situ de ces acides gras à partir des glucides du régime de gavage. Par ailleurs, compte tenu des conditions expérimentales, il paraît difficile de mettre en comparaison ces observations sur la composition en acides gras avec les travaux réalisés antérieurement chez l'oie (Leclercq et al, 1968) ou chez les 2 types de canards (Mulard et Barbarie) (Leclercq et Blum, 1975; Baldissera Nordio, 1975). En effet, outre la variabilité interspécifique observée, d'autres facteurs de variation qui n'ont pas été pris en compte dans cet essai peuvent intervenir (âge des animaux, conduites en élevage et en gavage). Dans le cas de notre expérimentation, sur les 2 facteurs étudiés, seul le format des animaux fait apparaître des différences de composition en acides gras hépatiques. La proportion plus importante en acide palmitique - premier acide gras issu de la synthèse de novo - observée chez les animaux de petit format, pourrait être le reflet d'une lipogénèse encore prépondérante. La conduite menée en gavage semble entraîner pour ces animaux une saturation au niveau des phénomènes di- 
gestifs. Par contre, les taux plus importants de C14:0, C14:1 et C16:1 observés chez les animaux les plus lourds pourraient traduire une lipolyse ou un état de remaniement post-digestif.

En conclusion, la perte de poids de foie observée entre $13 \mathrm{~h}$ et $19 \mathrm{~h}$ de jeûne, semble accompagner la déplétion du tractus digestif distal. Cette perte pondérale ne s'observe pas chez les animaux en phase digestive : la masse pondérale des animaux légers n'évoluant pas entre ces 2 stades de jeûne retenus.

Quel que soit l'état des animaux, on observe une détérioration de la qualité du foie estimée par le taux de fonte, sans qu'il soit possible néanmoins de mettre en évidence une différence de composition biochimique, et sans que cela se traduise par des profils d'acides gras différents.

Les foies des animaux les plus légers présentent une accumulation plus importante d'acide palmitique, d'eau tissulaire associée à une teneur plus faible en protéines, ce qui peut être relié à la saturation plus rapide de leurs capacités d'exportation. Cet état de saturation plus important en fin de gavage, consécutif à une différence de développement à l'âge de 10 semaines, semble influencer la nature des acides gras hépatiques.

\section{REMERCIEMENTS}

Nous tenons à remercier $C$ Vallèse, $H$ Manse et G Latil pour leur contribution technique lors de cette experimentation.

\section{RÉFÉRENCES}

Augustine PC (1982) Effect of feed water deprivation on organ and blood characteristics of young turkeys. Poultry Sci 61, 796-799

Babilé R (1989) La production de foies gras de canards de Barbarie (Caïrina moschata) : As- pects génétiques, nutritionnels et technologiques. Thèse Docteur État INPT, $315 p$

Babilé R, Auvergne A (1986) Qualité des foies gras : comparaison de différentes méthodes d'appréciation de la fonte lipidique. Évolution en fonction du temps. Rec Med Vét 162(2), 151-156

Babilé R, Auvergne A, Delpech P (1987a). Consommation de maïs au cours du gavage : incidence du gaveur, du type génétique et de l'âge. Rec Med Vet 163, 679-682

Babilé R, Auvergne A, Delpech P, Meirieu O (1987b) Évolution de la consommation de mais au cours du gavage et incidence sur la production de foie gras chez le canard de Barbarie. Ann Zootech 36, 73-84

Baldissera Nordio C (1975) Ricerche sulla qualità del fegato grasso di oche ed anatre iperalimentate con maïs. Avicoltura 3, 55-65

Burrin DG, Britton RA, Ferrel CL (1988) Visceral organ size and hepatocyte metabolic activity in fed and fasted rats. J Nutr 118, 1547-1552

Dagnélie P (1975) Théorie et méthodes statistiques. Les méthodes de l'inférence statistique, Vol 2, Presses agronomiques de Gembloux, Belgique

Folch J, Lees M, Sloane-Stanley CHS (1957) A simple method for the isolation and purification of total lipids from animal tissues. J Biol Chem 226, 497-509

Goodridge AG (1968) The effect of starvation and starvation followed by feeding on enzyme activity and the metabolism of ${ }^{\circ} 14-\mathrm{C}$ glucose in liver from growing chicks. Biochem J 108, 667-673

Goodridge AG, Ball EG (1968) Lipogenesis in the pigeon: in vitro studies. Am J Physiol $211,803-808$

Jensen LS, Cervantes HM, Takahashi K (1984) Liver lipid content in broilers as affected by time without feed or feed and water. Poultry Sci 63, 2404-2407

JOCE (1971a) J O Comm Eur, Dosage de l'humidité, L279/8

JOCE (1971b) J O Comm Eur, Dosage des cendres brutes, L155/20

Leclercq B, Blum JC (1975) Étude de la suralimentation forcée : effet sur le métabolisme hépatique et les formes de transport hépatiques chez le canard. Ann Biol Anim Biochim Biophys 15 (3), 559-568 
Leclercq B, de Carville H, Guy G (1989) Pertes de poids du canard de Barbarie mâle lors du jeûne avant l'abattage. INRA, Prod Anim 2 (5), 353-356

Leclercq B, Durand G, Delpech P, Blum JC (1968) Note préliminaire sur l'évolution des constituants biochimiques du foie au cours du gavage de l'oie. Ann Biol Anim Biochim Biophys 8, 549-556

Le Maho $Y$, Vu Van Kha $H$, Koubi $H$, Dewasmes G, Girard J, Ferré P, Cagnard M (1981) Body composition, energy expenditure, and plasma metabolites in long-term fasting geese. Am J Physiol 241, E342-E354

Leveillé GA, Romsos DR, Yeh YY, O'Hea EK (1975) Lipid biosynthesis in the chick. A consideration of site of synthesis, influence of diet and possible regulartory mechanisms. Poultry Sci 54, 1075-1093
Sibbald IR, Moorse PM (1983) The effects of feed input and excreta collection time on estimates of metabolic plus endogenous energy losses in the bioassay for true metabolizable energy. Poultry Sci 62, 68-76

Verdouw $H$, Van Echteld CJA, Dekkers EMJ (1977) Ammonium determination based on indophenol formation with sodium salicylate. Water Res 12, 399-402

Yeh YY, Leveillé GA, Willey JH (1970) Influence of dietary lipid on lipogenesis and on the activity of malic enzyme and citrate cleavage enzyme in liver of the growing chick. $J$ Nutr 100 , 917-924

Yeh YY, Leveillé GA (1971) In vitro and in vivo restoration of hepatic lipogenesis in fasted chicks. J Nutr 101, 803-810

WPSA (1984) Working Group $n^{\circ} 5$, JF Jensen ed, $33 \mathrm{p}$ 